\title{
Orbital stability of Earth-type planets in stellar binary systems
}

\author{
Jason Eberle, Manfred Cuntz and Zdzislaw E. Musielak
}

Department of Physics, University of Texas at Arlington, Arlington, TX, USA email: wjeberle@uta.edu,cuntz@uta.edu,zmusielak@uta.edu

\begin{abstract}
An important factor in estimating the likelihood of life elsewhere in the Universe is determining the stability of a planet's orbit. A significant fraction of stars like the Sun occur in binary systems which often has a considerable effect on the stability of any planets in such a system. In an effort to determine the stability of planets in binary star systems, we conducted a numerical simulation survey of several mass ratios and initial conditions. We then estimated the stability of the planetary orbit using a method that utilizes the hodograph to determine the effective eccentricity of the planetary orbit. We found that this method can serve as an orbital stability criterion for the planet.
\end{abstract}

\section{Introduction and method}

Based on observational evidence, a significant number of extrasolar planets are found in binary and multiple stellar systems, see Patience et al. 2002, Eggenberger et al. 2004, which is a strong motivation to pursue detailed theoretical studies of planetary orbital stability in stellar binaries. Previous results have been obtained by, e.g., Musielak et al. (2005), Cuntz et al. (2007), and Eberle et al. (2008). In the following, we present a new method that relies on a differential geometrical approach based on the analysis of the curvature of the hodograph in the synodic coordinate system. We found that when orbital instability occurs, the median of the eccentricity distribution exceeds unity. This criterion can be included in detailed simulations in an automated mode allowing the identification of planetary ejection in a straightforward manner. Intuitively, this criterion also agrees with the most basic property of conic sections representing closed orbits for $e<1$ and open orbits for $e \geqslant 1$, although it utilizes a modified definition of eccentricity (Eberle \& Cuntz 2010).

\section{Results and discussion}

We define $\mu=M_{2} / M$ with $M=M_{1}+M_{2}$, where $M_{1}$ and $M_{2}$ are the masses of the primary and secondary star, respectively. Furthermore, $\rho_{0}$ denotes the planet's relative initial distance from its host (primary) star. The mass of the planet is assumed to be very small compared to the stellar binary masses. If the planet orbits close to the primary star, the effective eccentricity remains low for the entire duration of the simulation. When the parameters are such that the zero velocity curve is nearly intersecting the L1 equilibrium point $\left(C_{1}\right.$ contour, green in Fig. 1), the effective eccentricity increases. For mass ratios less than 0.1 the zero velocity contour corresponding to L2 $\left(C_{2}\right.$, red in Fig. 1$)$ is a suitable limit for instability. For initial conditions where the planet is far enough away that the zero velocity curve as already opened up at L3 $\left(C_{3}\right.$ contour, black in Fig. 1), the planet is not well defined to be orbiting the primary star and quickly escapes the region of the binary stars and for most initial conditions, the planet quickly returns and rapidly 


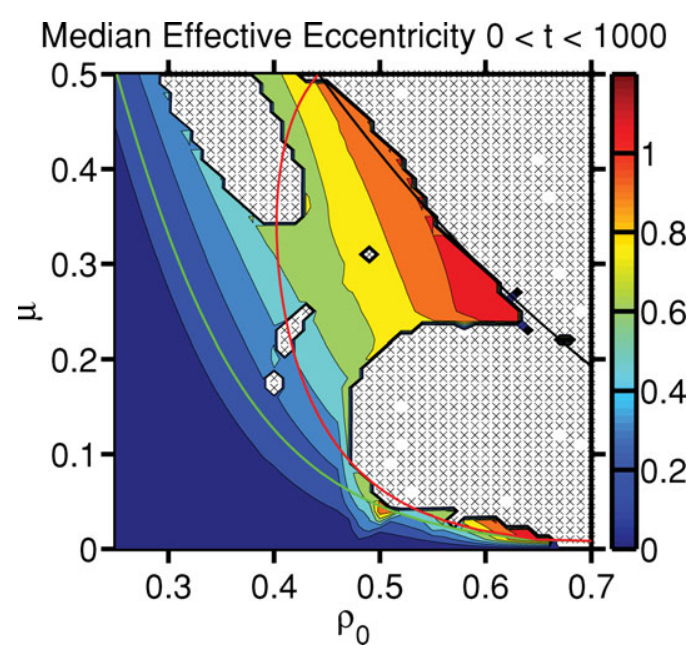

Figure 1. See text for details.

approaches one of the stars. The white regions with black crosses represent simulations that were terminated before the maximum 1000 year limit due to captures by one of the stars or ejection from the system. One unstable region is in the upper mass ratio range, roughly from $\mu=0.35$ to 0.50 and another is from 0.00 to 0.23 . In between these two regions $(\mu=0.24$ to 0.34$)$ is a quasi-stable region in which the effective eccentricity increases almost uniformly. The boundaries of this region remain basically the same for the duration of the simulation. Of course, we should not be surprised if the quasi-stable region were to shrink over a longer time period as 1000 orbits is not a very long time when considering long term stability. In fact, there is already a instability "island chain" slowly forming right in the middle of this quasi-stable region. The reasons for this instability "archipelago" are still under investigation.

\section{Conclusions}

A comparison with the zero-velocity contour of the planetary orbit shows that this criterion, which is a sufficient criterion for orbital instability, closely coincides with the opening of the zero-velocity contour at the Lagrange point L3, located to the right of the primary star, as discussed by Cuntz et al. (2007) and Eberle et al. (2008). If instability occurs, extremely large effective eccentricities are found, indicating a highly hyperbolic planetary orbit in the synodic coordinate system, a stark indication of planetary escape. Although our results have been obtained for the special case of the restricted three-body problem, we expect that it may also be possible to augment this criterion to planets in generalized stellar binary systems, and to use it concerning long-term simulations.

\section{References}

Cuntz, M., Eberle, J., \& Musielak, Z. E. 2007, ApJ(Letters) 669, L105

Eberle, J., Cuntz, M., \& Musielak, Z. E. 2008, A\&A 489, 1329

Eberle, J. \& Cuntz, M. 2010, A\&A, submitted

Eggenberger, A., Udry, S., \& Mayor, M. 2004, A\&SA 417, 353

Musielak, Z. E., Cuntz, M., Marshall, E. A., \& Stuit, T. D. 2005, A\&6A 434, 355 \& Erratum 2008, A\&A 480, 573

Patience, J., White, R. J., Ghez, A. M. et al. 2002, ApJ 581, 654 